\title{
BRIEF COMMUNICATION OPEN Clinical assessment of speech correlates well with lung function during induced bronchoconstriction
}

\author{
Nicholas Tayler $^{1}$, Christopher Grainge ${ }^{1,2}$, Kerry Gove ${ }^{1}$, Peter Howarth ${ }^{1,3}$ and Judith Holloway ${ }^{1}$
}

Clinical assessment of asthma often includes a crude assessment of speech, for example whether the patient can speak in full sentences. To date, this statement, despite appearing in national asthma guidelines, has not been related to lung function testing in asthma exacerbation. Seven asthmatics underwent a bronchial challenge and were then recorded reading a standardised text for $1 \mathrm{~min}$. The recordings were played to 88 healthcare professionals who were asked to estimate $\mathrm{FEV}_{1} \%$ predicted. Health care professionals' estimations showed moderate correlation to $\mathrm{FEV}_{1} \%$ predicted (rho $=0.61 P<0.01$ ). There were no significant differences between professionals grouped by seniority or speciality. Speech can intuitively be estimated by health care professionals with moderate accuracy. This gives an evidence basis for the assessment in speech in acute asthma and may provide a new avenue for monitoring.

npj Primary Care Respiratory Medicine (2015) 25, 15006; doi:10.1038/npjpcrm.2015.6; published online 26 February 2015

\section{INTRODUCTION}

Asthma is a chronic inflammatory respiratory condition that is characterised by bronchoconstriction leading to a multitude of symptoms, including wheeze, cough and dyspnoea. ${ }^{1}$

Assessment and monitoring of asthma is multifaceted, including symptom-based diaries, ${ }^{2}$ as well as objective measurements of lung function and airway inflammation. Clinicians may also evaluate the way the patient is speaking, either consciously or subconsciously, as part of an assessment of asthma severity. ${ }^{3}$

Speech assessment is not a formal technique; however, statements recorded in patient's notes such as 'inability to complete sentences in one breath' are used with other measures to triage patients into severity categories during acute asthma in adults and children in current published guidelines. ${ }^{4,5}$ In addition, asthma limiting speech has been used as an indicator of severity in longitudinal questionnaire-based studies of asthma. ${ }^{6}$ To our knowledge, it has not been determined whether subjective assessment of changes in the quality of speech in patients with asthma can be related to alterations in pulmonary function.

\section{Hypothesis}

Health care professionals are able to accurately estimate forced expiratory volume in $1 \mathrm{~s}\left(\mathrm{FEV}_{1}\right)$ during experimentally induced bronchoconstriction from speech samples recorded at the time of bronchial challenge.

\section{MATERIALS AND METHODS}

Adult mild asthmatics atopic to house dust mite (Dermatophagoides pteronyssinus) taking short-acting $\beta_{2}$-agonists as required as their only asthma therapy (Global Initiative for Asthma step 1) were recruited. All the participants gave written informed consent, and the study was approved by the local research ethics committee. The participants were excluded if they had any past medical history of psychiatric issues such as anxiety or haematological anomalies such as anaemia, as these factors may have influenced baseline speech quality.

Seven volunteers were recruited and underwent either a methacholine or allergen ( $D$. pternyssinus) challenge to a targeted fall in $\mathrm{FEV}_{1}$ of $20 \%$ from baseline according to published protocols. $^{7,8}$ After each challenge dose, the participant read a standardised text for $30 \mathrm{~s}$ into a digital recorder fitted with an external microphone set at $10 \mathrm{~cm}$ from the mouth (Olympus DM450 Speech Recorder with Olympus ME34 Microphone, Tokyo, Japan).

The patients also evaluated their symptoms on a $200-\mathrm{mm}$ horizontal visual analogue scale (VAS) from 'no asthma symptoms' to 'worst possible asthma symptoms' immediately after each episode of speech had been recorded. One speech track from each participant was then selected to display a range of lung functions from $100 \% \mathrm{FEV}_{1}$ predicted to $53 \% \mathrm{FEV}_{1}$ predicted; the quality of speech was not known before selection of the data files. Predicted lung function was calculated using the European Respiratory Society data. ${ }^{9}$

Recorded speech was played on standardised equipment to 88 individuals including laboratory scientists, clerical support staff, nurses and physicians from a range of specialities including respiratory medicine, family practice, allergy medicine and paediatrics.

Before hearing the speech recordings, assessors were read a standard introduction (see Supplementary Information S1) briefly explaining what $\mathrm{FEV}_{1}$ means and how speech samples were collected. They then listened to the speech recordings and estimated $\mathrm{FEV}_{1}$ on a $150-\mathrm{mm}$ horizontal VAS-labelled ' $\mathrm{FEV}_{1} 100 \%$ predicted' to ' $F E V_{1} 0 \%$ predicted'. The VAS used was a continuous line with no incremental markings.

\footnotetext{
${ }^{1}$ University of Southampton Faculty of Medicine, Southampton General Hospital, Southampton, UK; ${ }^{2}$ Department of Respiratory and Sleep Medicine, Hunter Medical Research Institute, John Hunter Hospital, The University of Newcastle, New Lambton, NSW, Australia and ${ }^{3}$ Southampton NIHR Respiratory Biomedical Research Unit, Southampton Centre for Biomedical Research, University Hospital Southampton NHS Foundation Trust, Southampton, UK.

Correspondence: N Tayler (njt1g09@soton.ac.uk)
}

Received 28 August 2014; revised 29 October 2014; accepted 2 December 2014 


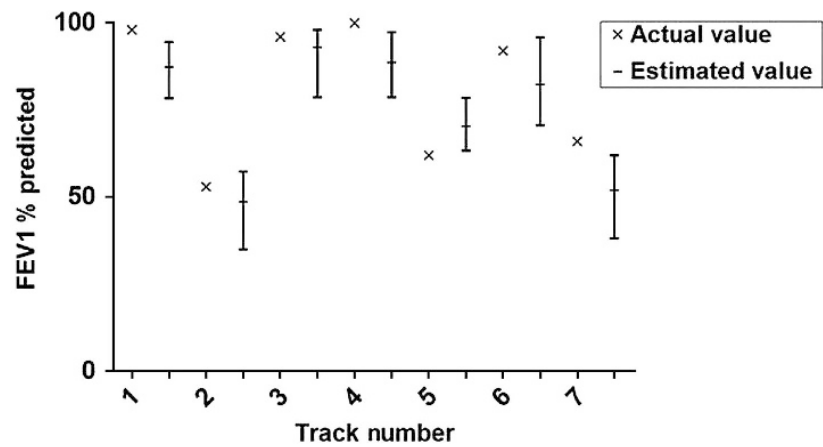

Figure 1. Actual and median estimated values of $\mathrm{FEV}_{1} \%$ predicted. Figure shows the $\mathrm{FEV}_{1} \%$ predicted of each recording, accompanied by the median estimated value and interquartile range for each

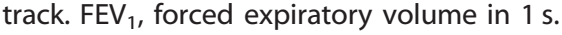

Table 1. Correlation of $\mathrm{FEV}_{1}$ estimated from speech compared with actual $\mathrm{FEV}_{1}$ by speciality or clinical experience of assessor

\begin{tabular}{lcccc}
\hline Speciality or experience & $\mathrm{N}$ & $\begin{array}{c}\text { Correlation } \\
\text { (rho) }\end{array}$ & P value & $\begin{array}{c}\text { Number } \\
\text { of tracks }\end{array}$ \\
\hline Allergy and dermatology & 6 & 0.58 & $<0.01$ & 42 \\
General practice & 15 & 0.62 & $<0.01$ & 105 \\
Medical & 5 & 0.64 & $<0.01$ & 35 \\
Paediatrics & 15 & 0.68 & $<0.01$ & 105 \\
Respiratory & 35 & 0.57 & $<0.01$ & 245 \\
Surgical & 7 & 0.64 & $<0.01$ & 49 \\
Scientist & 5 & 0.74 & $<0.01$ & 35 \\
1=Consultant, matron & 27 & 0.61 & $<0.01$ & 189 \\
2= Registrar, advanced nurse & 9 & 0.64 & $<0.01$ & 63 \\
3=SHO, CNS & 16 & 0.55 & $<0.01$ & 112 \\
4=FY1, staff nurse & 28 & 0.62 & $<0.01$ & 196 \\
5= Non-clinical & 6 & 0.67 & $<0.01$ & 42 \\
\hline
\end{tabular}

The correlation coefficients for each group of health care professionals in the top section, and for the same professionals grouped by experience category in the bottom section.

Abbreviations: CNS, clinical nurse specialist; FY1-, foundation year 1 doctor; $\mathrm{SHO}$, senior house officer.

No significant difference in correlation was found between specialities $(P=0.29)$ or clinical experience groups $(P=0.39)$ when analysed with one-way analysis of variance.

\section{RESULTS}

Participants' symptoms measured by VAS strongly correlated with lung function ( $r h o=0.86 P=0.014$ ).

Estimated $\mathrm{FEV}_{1}$ from listening to speech showed a moderate correlation with measured $\mathrm{FEV}_{1}$ values (rho $=0.61, P<0.01$ ). The median absolute difference of assessor's estimation compared with measured $\mathrm{FEV}_{1}$ was $10.6 \%$ (interquartile range $4-21.3 \%$ ). This is shown in Figure 1.

There was no significant correlation between the patient's $\mathrm{FEV}_{1} \%$ predicted and the median difference of $\mathrm{FEV}_{1}$ estimation $(P=0.43)$.

No significant difference was found in the ability to estimate $\mathrm{FEV}_{1}$ from speech between assessors when grouped either by clinical experience or specialisation $(P=0.39$ and $P=0.29)$. This is summarised in Table 1.

\section{DISCUSSION}

Health care professionals, regardless of clinical experience or speciality, can estimate lung function with moderate accuracy on the basis of recorded speech alone with a median error of $10.6 \%$.
In addition, asking our volunteers to evaluate their symptoms using a VAS also provided a reasonably accurate assessment of $\mathrm{FEV}_{1}$. Assessment of speech is a reasonable part of a comprehensive asthma evaluation, and it may be especially useful when lung function testing is not possible or available, although caution should be taken not to overestimate asthma severity, as seen in the majority of our results. This may be due to a confounding factor, as health care professionals were aware that the patients were asthmatic, and thus may have subconsciously adjusted their estimations towards worsening lung function.

There was no significant correlation between $\mathrm{FEV}_{1}$ values and absolute difference of estimation, indicating that the accuracy of prediction is not affected by pulmonary function.

Although assessment of speech has been advocated in internationally approved asthma guidelines for many years $\mathrm{s}^{4,5}$ and has been used in long-term follow-up of asthma, ${ }^{10}$ the ability of health care professionals to estimate lung function from quality of speech has not previously been assessed. We demonstrate that alterations in speech that occur with worsening asthma can be detected and apparently intuitively quantified, as type and length of training do not affect accuracy.

More precise determination of the changes being detected, for example, wheeze and duration or frequency of breath sounds, could open new avenues for non-invasive monitoring of asthma. In addition, further work could include determining whether categorised exacerbations of asthma (mild, moderate, severe, life threatening) can be detected with suitable accuracy by speech analysis. Repeating this study with a larger sample size of speech tracks from asthmatics could improve results.

In the meantime, traditional assessment of asthma severity by evaluation of speech has value and should continue to be recorded.

\section{ACKNOWLEDGEMENTS}

The authors thank the Southampton Biomedical Research Unit nurses, all the patients and health care professionals for their participation.

\section{CONTRIBUTIONS}

All authors designed the study, collected and analysed the data and wrote the manuscript.

\section{COMPETING INTERESTS}

The authors declare no conflict of interest.

\section{FUNDING}

The study was funded by the Asthma, Allergy and Inflammation Research Charity, Southampton, UK.

\section{REFERENCES}

1 McCormack MC, Enright PL. Making the diagnosis of asthma. Respir Care 2008; 53 583-590; discussion 590-592.

2 Meltzer EO, Busse WW, Wenzel SE, Belozeroff V, Weng HH, Feng J et al. Use of the Asthma Control Questionnaire to predict future risk of asthma exacerbation J Allergy Clin Immunol 2011; 127: 167-172.

3 Bell D, Layton A, Gabbay J. Use of a guideline based questionnaire to audit hospital care of acute asthma. BMJ 1991; 302: 1440-1443.

4 British Thoracic Society, Scottish Intercollegiate Guidelines Network (BTS, SIGN). British Guideline on the Management of Asthma- a national clinical guideline 2012. Available at www.sign.ac.uk/guidelines/fulltext/.

5 National Asthma Council Australia Australian Asthma Handbook - Quick Reference Guide, Version 1.0, 2014. Available from www.asthmahandbook.org.au (accessed 26 May 2014). 
6 Lai C, Beasley R, Crane J, Foliaki S, Shah J, Weiland S. Global variation in the prevalence and severity of asthma symptoms: Phase Three of the International Study of Asthma and Allergies in Childhood (ISAAC). Thorax 2009; 64: 476-483.

7 Schulze J, Rosewich M, Riemer C. Methacholine challenge-comparison of an ATS protocol to a new rapid single concentration technique. Respir Med 2009; 103: 1898-1903.

8 Grainge CL, Lau LCK, Ward JA, Dulay V, Lahiff G, Wilson S et al. Effect of bronchoconstriction on airway remodeling in asthma. N Engl J Med 2011; 364: 2006-2015.

9 Roca J, Burgos F, Sunyer J, Saez M, Chinn S, Antó JM et al. References values for forced spirometry. Eur Respir J 1998; 11: 1354-1362.
10 Rosier MJ, Bishop J, Nolan T, Robertson CF, Carlin JB, Phelan PD. Measurement of functional severity of asthma in children. Am J Respir Crit Care Med 1994; 149: 1434-1441.

(C) This work is licensed under a Creative Commons AttributionNonCommercial-ShareAlike 4.0 International License. The images or other third party material in this article are included in the article's Creative Commons license, unless indicated otherwise in the credit line; if the material is not included under the Creative Commons license, users will need to obtain permission from the license holder to reproduce the material. To view a copy of this license, visit http:// creativecommons.org/licenses/by-nc-sa/4.0/

Supplementary Information accompanies the paper on the npj Primary Care Respiratory Medicine website (http://www.nature.com/npjpcrm) 